\title{
Dielectric mismatch effect on shallow impurity states in a semiconductor nanowire
}

\author{
Bin Li, ${ }^{1}, *$ A. F. Slachmuylders, ${ }^{1}$ B. Partoens, ${ }^{1}$ W. Magnus,,${ }^{1,2}$ and F. M. Peeters ${ }^{1, \dagger}$ \\ ${ }^{1}$ Departement Fysica, Universiteit Antwerpen, Groenenborgerlaan 171, B-2020 Antwerpen, Belgium \\ ${ }^{2}$ Interuniversity Microelectronics Centre, Kapeldreef 75, B-3001 Leuven, Belgium \\ (Received 3 January 2008; revised manuscript received 20 February 2008; published 19 March 2008)
}

\begin{abstract}
Shallow impurity states in a freestanding semiconductor nanowire and in a semiconductor nanowire surrounded by a metallic gate are studied within the effective mass approximation. We calculate the total energy of the electron and the binding energy by using (1) a variational approach, which provides an upper bound to the electron energy, and (2) the finite element method which is "numerically" exact. The dependence of the binding energy and the extent of the shallow impurity wave function on the wire radius $R$ and the ionized impurity position in the nanowire is examined. The validity of the often used variational calculation is critically examined by calculating the difference of the binding energies obtained from the two different methods as a function of the wire radius $R$ and the ionized impurity position.
\end{abstract}

DOI: 10.1103/PhysRevB.77.115335

PACS number(s): 73.22.-f, 73.21.-b, 77.22.-d, 61.46.Km

\section{INTRODUCTION}

Semiconductor nanowires (NWs) are attractive building blocks for nanoscale electronic devices, including field-effect transistors,,${ }^{1,2}$ inverters, ${ }^{3}$ logic circuits, ${ }^{7}$ sensors, ${ }^{8-10}$ and decoders,,${ }^{11}$ because of the intrinsic small size and the promise of enhanced mobility. Continued progress toward integrated nanoelectronic circuits will require advances in our ability to better control the electronic properties of these building blocks and to assemble them into increasingly complex structures. Considerable efforts have been placed on doping $\mathrm{Si}^{3-5}{ }^{3-5 e},{ }^{6,12}$ and GaN NWs to control their electrical properties. However, doping of nanostructures remains a challenge as a result of both fundamental synthetic issues and statistical fluctuations that are intrinsic to homogeneous doping of small structures. Moreover, charged dopant centers will limit the mobility ${ }^{20}$ and the corresponding performance of these semiconductor nanowires.

The composition of the nanowires can also be modulated along the radial plane to form core-shell structures. ${ }^{13-17}$ Due to the dielectric and strain mismatch at the core-shell interface, the properties of the axial nanowires will be modified. The effect of the dielectric confinement to the electronic structures, ${ }^{18,19}$ scattering properties, ${ }^{20,21}$ and excitonic absorption spectrum ${ }^{22-24}$ of free electrons in freestanding nanostructures have been investigated. The electronic structures of a core-shell wire, which take strain, piezoelectric, and dielectric effects into account, have been calculated using the tight-binding method. ${ }^{25}$ The localization of the electron states in a nanowire surrounded by a metallic shell nanocontact ${ }^{26}$ was also recently investigated.

In this paper, we investigate the effect of the dielectric mismatch on the shallow impurity states in semiconductor nanowires and we study how these bound states depend on the radial position of the impurity. We perform such a calculation for a freestanding NW and a NW surrounded by a metallic gate. We will use two independent methods: the variational method and the finite element method (FEM) within the effective mass approximation. ${ }^{27,28}$ The dependence of the binding energies and wave functions on the wire radius $R$ and the impurity radial position $\rho_{i}$ will be investigated.
This paper is organized as follows: We present the physical model in Sec. II. The results of the variational and finite element calculations are presented in Secs. III and IV, respectively. The validity of the variational calculation is discussed in Sec. V. Our conclusions are presented in Sec. VI.

\section{MODEL}

A schematic illustration of the model system is shown in Fig. 1. We used the effective mass approximation, where the core material has an effective mass $m_{e}^{*}$ and dielectric permittivity $\varepsilon_{1}$, while the surrounding barrier material has a dielectric permittivity $\varepsilon_{2}$. The potential at an arbitrary point inside such a system is the sum of the potential generated by the electron and the ionized impurity. A similar problem was previously studied in Ref. 22, where the authors calculated the binding energies and excitonic absorption spectrum of a freestanding GaAs nanowire. Here, the potential at an arbitrary point generated by a single charged particle at $\overrightarrow{r_{q}}$ can be obtained by solving the following Poisson equation: ${ }^{20,22}$

$$
\nabla\left(\varepsilon(\rho) \nabla V\left(\vec{r}, \overrightarrow{r_{q}}\right)\right)=-q \delta\left(\vec{r}-\overrightarrow{r_{q}}\right),
$$

with

$$
\varepsilon(\rho)=\varepsilon_{2}+\left(\varepsilon_{1}-\varepsilon_{2}\right) \theta(R-\rho) .
$$

So the potential at an arbitrary point inside the wire for a single charged particle is ${ }^{18}$

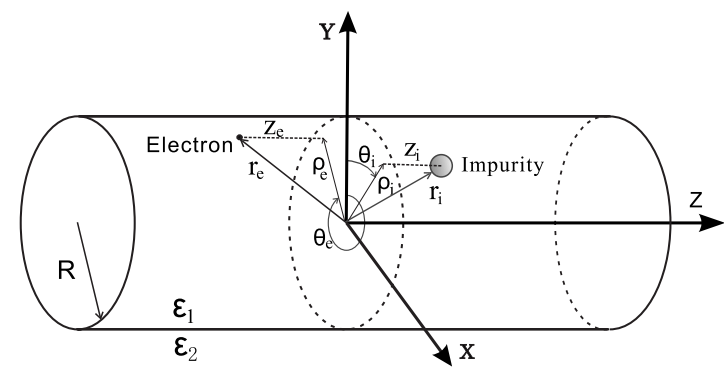

FIG. 1. Schematics of the system: A shallow impurity in a nanowire surrounded by a different dielectric medium. 


$$
\begin{aligned}
V_{\text {in }}\left(\vec{r}, \vec{r}_{q}\right) & \\
= & \frac{e}{\varepsilon_{1}\left|\vec{r}-\vec{r}_{q}\right|}-\frac{2 e}{\pi \varepsilon_{1}} \sum_{m=-\infty}^{+\infty} \int_{0}^{+\infty} d k \\
& \times\left[e^{i m\left(\theta-\theta^{\prime}\right)} \cos \left[k\left(z-z_{q}\right)\right] f_{m}\left(\frac{\varepsilon_{1}}{\varepsilon_{2}}, k R\right) I_{m}(k \rho) I_{m}\left(k \rho_{q}\right)\right],
\end{aligned}
$$

where

$$
f_{m}\left(\frac{\varepsilon_{1}}{\varepsilon_{2}}, k R\right)=\frac{\left(1-\frac{\varepsilon_{1}}{\varepsilon_{2}}\right) K_{m}(k R) K_{m}^{\prime}(k R)}{I_{m}(k R) K_{m}^{\prime}(k R)-\frac{\varepsilon_{1}}{\varepsilon_{2}} I_{m}^{\prime}(k R) K_{m}(k R)},
$$

with $K_{m}(x)$ and $I_{m}(x)$ the modified Bessel functions of the third and first kind, respectively, and $K_{m}^{\prime}(x)$ and $I_{m}^{\prime}(x)$ their derivatives. The electron potential energy is obtained after taking the following integral:

$$
U_{t o t}\left(\vec{r}_{e}\right)=-e \int d \vec{r} \delta\left(\vec{r}-\vec{r}_{e}\right)\left[V\left(\vec{r}, \vec{r}_{e}\right)+V\left(\vec{r}, \vec{r}_{i}\right)\right] .
$$

The first term in the integral is the electrostatic energy due to the images of the electron itself. We should subtract the electrostatic self-energy coming from part of the first term that is proportional to $\delta\left(\vec{r}-\vec{r}_{e}\right) /\left|\vec{r}-\vec{r}_{e}\right|$. The second term is the electrostatic energy due to the ionized impurity and its images. Then the electrostatic energy of the electron can be rewritten as $^{18}$

$$
U_{\text {tot }}\left(\vec{r}_{e}\right)=U_{\text {dir }}\left(\vec{r}_{e}, \vec{r}_{i}\right)+U_{\text {ind }}\left(\vec{r}_{e}, \vec{r}_{i}\right)+U_{\text {self }}\left(\rho_{e}\right),
$$

which consists of three parts: the direct Coulomb potential energy $U_{d i r}\left(\vec{r}_{e}, \vec{r}_{i}\right)$; the induced potential energy $U_{\text {ind }}\left(\vec{r}_{e}, \vec{r}_{i}\right)$, which comes from the electron and the image of the ionized impurity; and the self potential energy $U_{\text {self }}\left(\rho_{e}\right)$, which is the interaction between the electron and its image. ${ }^{18}$ Moreover, the self potential energy $U_{\text {self }}\left(\rho_{e}\right)$ is independent of the ionized impurity position and depends only on the electron radial coordinate. We can rewrite $U_{\text {tot }}$ as follows:

$$
U_{t o t}=U_{i n t}\left(\vec{r}_{e}, \vec{r}_{i}\right)+U_{\text {self }}\left(\rho_{e}\right)
$$

with $U_{i n t}\left(\vec{r}_{e}, \vec{r}_{i}\right)$ the electrostatic potential energy between the electron and the ionized impurity together with its images.

Then the Schrödinger equation of the electron inside the nanowire within the effective mass approximation is given by

$$
\left(-\frac{\hbar_{2}}{2 m_{e}^{*}} \nabla_{e}^{2}+U_{t o t}+U_{c}\left(\rho_{e}\right)\right) \psi_{e}=E_{t o t} \psi_{e},
$$

where $U_{c}\left(\rho_{e}\right)$ is the confinement potential, which we assume to be a circular potential well with infinite high barriers as follows:

$$
U_{c}\left(\rho_{e}\right)= \begin{cases}0, & \rho_{e} \leq R \\ \infty, & \rho_{e}>R .\end{cases}
$$

As we cannot solve the three-dimensional (3D) Schrödinger equation analytically, we will use two independent methods to solve this differential equation: variational method and FEM.

\section{RESULTS OF THE VARIATIONAL CALCULATION}

We assume that the radius of the wire is sufficiently small such that the single particle confinement energy is much larger than the Coulomb energy. This allows us to use the adiabatic approximation. We take the wave function in the radial plane to be the wave function as obtained by solving the two-dimensional (2D) circular infinite high well. ${ }^{28} \mathrm{Next}$, we multiply each side of the Schrödinger equation with the $(\rho, \theta)$ part of the wave function, averaging out the $(\rho, \theta)$ motion, which leads us to a one-dimensional effective Schrödinger equation in the $z$ direction.

Thus, we assume a factorized form of the wave function $\psi_{e}$ as $\varphi\left(\rho_{e}, \theta_{e}\right) \phi\left(z_{e}\right)$, multiply Eq. (7) with the normalized $\varphi^{*}\left(\rho_{e}, \theta_{e}\right)$ from the left, and integrate over the radial coordinates. Finally, we obtain the effective one-dimensional Schrödinger equation:

$$
\left(-\frac{\hbar^{2}}{2 m_{e}^{*}} \nabla_{z}^{2}+U_{e f f}(z)\right) \phi\left(z_{e}\right)=E_{z} \phi\left(z_{e}\right),
$$

with

$$
\begin{gathered}
E_{z}=E_{t o t}-E_{\rho}-E_{\text {self }}, \\
U_{e f f}(z)=\int_{0}^{R} \rho_{e} d \rho_{e} \int_{0}^{2 \pi} d \theta_{e} \varphi^{*}\left(\rho_{e}, \theta_{e}\right) U_{i n t}\left(\vec{r}_{e}, \vec{r}_{i}\right) \varphi\left(\rho_{e}, \theta_{e}\right),
\end{gathered}
$$

and

$$
E_{\text {self }}=\int_{0}^{R} \rho_{e} d \rho_{e} \int_{0}^{2 \pi} d \theta_{e} \varphi^{*}\left(\rho_{e}, \theta_{e}\right) U_{\text {self }}\left(\overrightarrow{r_{e}}\right) \varphi\left(\rho_{e}, \theta_{e}\right) .
$$

Here, $E_{\rho}=\left(\hbar x_{n}^{(l)}\right)^{2} / 2 m_{e}^{*} R^{2}$ is the confinement energy ${ }^{28}$ of the electron, which can be obtained by solving the 2D circular infinite high well. $x_{n}^{(l)}$ is the $n$th zero of the Bessel function of order $l$.

Since we are dealing with a shallow impurity that is similar to a hydrogen atom, we will use normalized hydrogenictype ground and first excited trial wave functions $\phi_{g}\left(z_{e}\right)$ $=(2 / \pi \beta)^{1 / 4} e^{-z_{e}^{2} / \beta}$ and $\phi_{f}\left(z_{e}\right)=2(2 / \pi)^{1 / 4} \beta^{-3 / 4} z_{e} e^{-z_{e}^{2} / \beta}$ along the $z$ direction (here, $\beta$ is the trial parameter). Then the ground and first excited state energies of the electron in the $z$ direction can be written in the following form:

$$
\begin{gathered}
E_{g}(\beta)=\frac{\hbar^{2}}{2 m_{e}^{*} \beta}+\frac{4}{\sqrt{2 \pi \beta}} \int_{0}^{+\infty} d z_{e} e^{-2 z_{e}^{2} / \beta} U_{e f f}(z) \\
E_{f}(\beta)=\frac{3 \hbar^{2}}{2 m_{e}^{*} \beta}+\frac{8 \sqrt{2}}{\beta^{3 / 2} \sqrt{\pi}} \int_{0}^{+\infty} d z_{e} z_{e}^{2} e^{-2 z_{e}^{2} / \beta} U_{e f f}(z)
\end{gathered}
$$

By minimizing Eqs. (10) and (11) with respect to $\beta$, we obtain the ground and first excited state energies of the elec- 


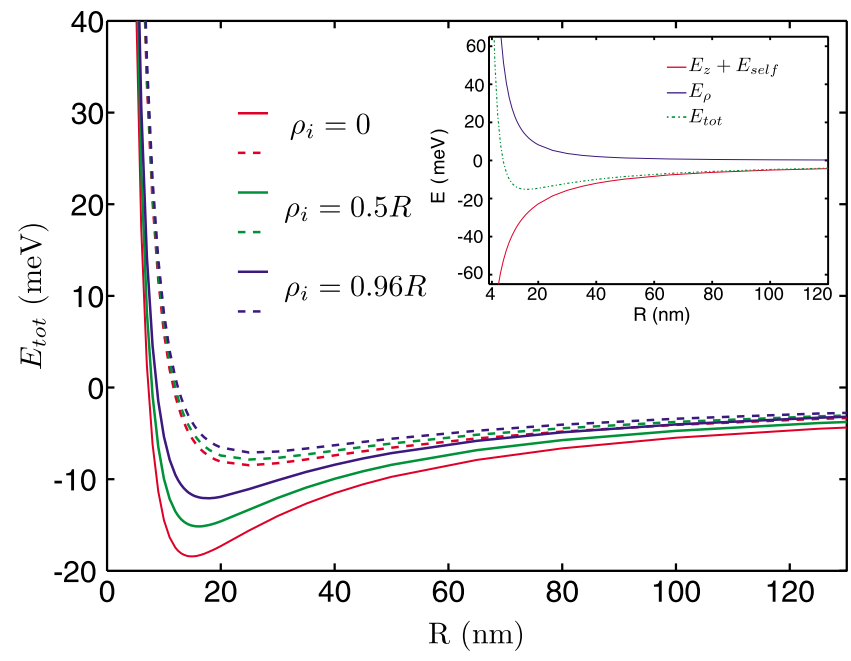

FIG. 2. (Color online) Ground state (solid line) and first excited state (dashed line) energies as a function of the wire radius $R$ in a freestanding GaAs nanowire. The ionized impurity is fixed at the center of the wire (red line), $0.5 R$ from the center (green line), and $0.96 R$ from the center (blue line). The inset shows the different contributions to the total energy as a function of the radius $R$ of the $\mathrm{NW}, \rho_{i}=0.5 R$.

tron in the $z$ direction, respectively. Here, we will consider two cases: a freestanding NW and a semiconductor wire surrounded by a metallic gate.

\section{A. Freestanding nanowire}

As an example, we consider a GaAs nanowire. For GaAs freestanding nanowires, we have $\varepsilon_{1}=12.5 \varepsilon_{0}, \varepsilon_{2}=\varepsilon_{0}$, and $m_{e}^{*}=0.067 m_{e}$. The ground state and first excited state energies for different values of wire radius $R$ are shown in Fig. 2 for the impurity fixed at the center of the wire, $0.5 R$ from the center, and $0.96 R$ from the center. We notice that the ground state and first excited state energies first decrease with increasing radius of the wire and, after reaching a local minimum, it will increase and asymptotically reach the 3D result. This behavior can physically be understood as follows. We can write the total energy as

$$
E_{\text {tot }}=E_{\rho}+E_{z}+E_{\text {self }} \text {. }
$$

The confinement energy $E_{\rho}$ is positive and proportional to $1 / R^{2}$. It decreases with increasing radius $R$ and is dominant for small $R$. While $E_{z}$ and $E_{\text {self }}$ are both proportional to $1 / R$ and the sum of these two terms is negative, it (the sum) increases when the radius $R$ increases and leads to the dominant contribution for large $R$. We make these tendencies clearer in the inset of Fig. 2: $E_{\rho}$ is very large when $R$ is small and decreases very quickly to zero, while $E_{z}+E_{\text {self }}$ will increase toward zero slower. So with increasing $R$, the total energy will first decrease as $E_{\rho}$, and then increase as the sum of the last two terms. When the ionized impurity is located $0.5 R$ from the axis of the wire, the total energy will reach its minimum value when $R$ is around $16 \mathrm{~nm}$. Figure 2 also shows that the total energy will be larger when the ionized

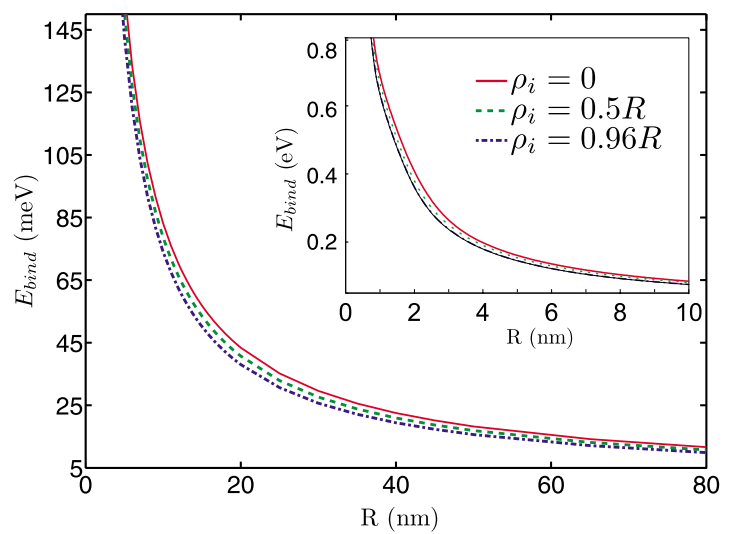

FIG. 3. (Color online) Binding energy as function of wire radius $R$. Inset: Zoom of the binding energy for small values of $R$.

impurity is closer to the boundary of the NW; however, for the case of freestanding nanowires, these differences are not very large.

Next, we will study the behavior of the binding energy with respect to the wire radius $R$. In the variational calculation, when there is no impurity inside the wire, the total energy of the electron is just the sum of the confinement energy $E_{\rho}$ and the self-energy $E_{\text {self }}$ [see Eq. (9), the selfenergy is only a function of the radius $R$ ]. So the binding energy is the difference between the total energy without an impurity and the total energy with an impurity, which is just $-E_{z}$. Figure 3 shows the dependence of the binding energy on the wire radius $R$ for three different ionized impurity positions. The binding energy increases when the radius $R$ becomes smaller and, due to the dielectric mismatch, the binding energy will be several hundreds of $\mathrm{meV}$ or even close to $1 \mathrm{eV}$ when the radius is several nanometers, as shown in the inset (similar results were presented in Refs. 19 and 24). The binding energies are also affected by the ionized impurity position: the closer the ionized impurity is to the edge of the NW, the smaller the binding energy will be. However, the differences in the binding energies are only noticeable when the radius is neither too large nor very small.

\section{B. Semiconductor nanowire surrounded by a metallic gate}

The metallic gate is modeled as having a dielectric permittivity $\varepsilon_{2} \approx+\infty$. Figure 4 shows the ground state and first excited state energies as a function of wire radius $R$ for three different ionized impurity positions. From Fig. 4, we notice that the main difference with freestanding nanowires in vacuum is that the total energy is much smaller. The curve for the ground state with the impurity fixed at $0.96 R$ overlaps with the curves of the first excited state. From the inset of Fig. 4, we see that the energies of the first excited state and the ground state for $\rho_{i}=0.96 R$ almost overlap with the curve of $E_{\rho}+E_{\text {self }}$, which is, in fact, the total energy of the electron inside the wire without an impurity. That means that the impurity has a small effect on the total energy and, consequently, the binding energy will be very small. This can also be seen from the curves of the binding energy shown in Fig. 5. 


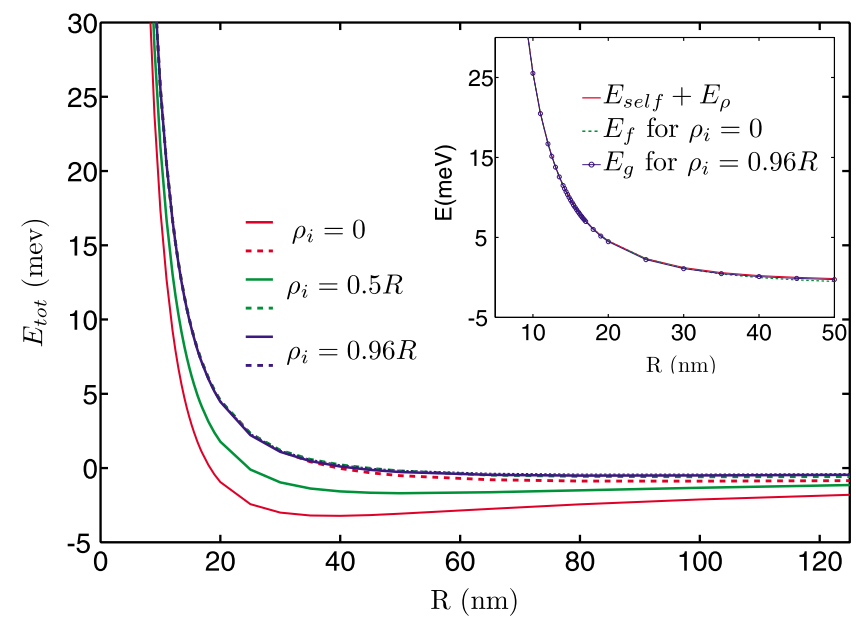

FIG. 4. (Color online) Ground state (solid lines) and first excited state (dashed lines) energies of a GaAs wire surrounded by a metallic gate for three different positions of the ionized impurity. Inset: Total energy of the electron inside the wire without an impurity compared with the total energy with an impurity.

When comparing Figs. 5 and 3, we notice that the impurity position influences much more strongly the binding energy for a GaAs wire surrounded by a metallic gate and that the impurity binding energy is much smaller than for the corresponding freestanding nanowire, even for a very small radius. For example, for $R=20 \mathrm{~nm}$ and $\rho_{i}=0$, the binding energy is about $45 \mathrm{meV}$ for a freestanding GaAs nanowire and $6 \mathrm{meV}$ for a GaAs nanowire with a metallic gate, and for $\rho_{i}=0.5 R$, the binding energies are 40 and $3 \mathrm{meV}$, respectively. This is due to the screening of the Coulomb potential by the metallic gate. This is also the reason why the impurity position has a large effect on the binding energy. The binding energy will decrease toward zero when the impurity moves from the center of the wire to the edge of the wire.

\section{NUMERICALLY “EXACT” RESULTS}

By using the technique of finite elements, we are able to solve the 3D Schrödinger equation numerically. In order to make the calculation faster, we will rewrite the $3 \mathrm{D}$

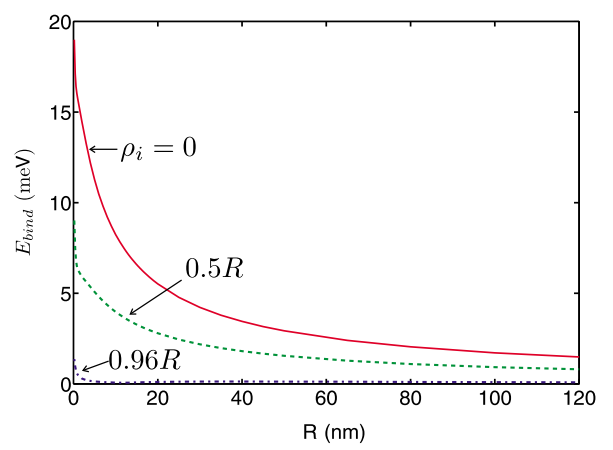

FIG. 5. (Color online) Binding energy as a function of the wire radius $R$ for different impurity positions in a NW surrounded by a metallic gate.

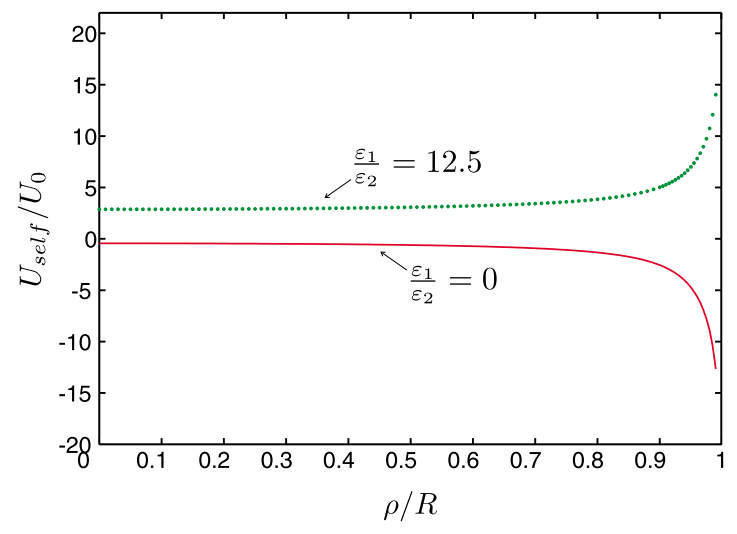

FIG. 6. (Color online) Self potential energy for a freestanding GaAs nanowire (green dotted curve) and a GaAs wire surrounded by a metallic gate (red solid curve) as a function of the electron radial position $\rho$. The energy is in units of $U_{0}=e^{2} / 4 \pi \varepsilon_{1} R$.

Schrödinger equation (7). The self potential energy that appears in the total electrostatic potential energy $U_{t o t}$ of the electron (6) will first be calculated and fitted to a function of the electron radial coordinate. The first term in Eq. (6) will be obtained by using a Poisson solver, which will give the electrostatic energy between the electron and the ionized impurity (together with the images of the ionized impurity). The 3D Schrödinger equation is then solved by using the finite elements method. Thus, the 3D Schrödinger equation is rewritten as

$$
\left(-\frac{\hbar^{2}}{2 m_{e}^{*}} \nabla_{e}^{2}+U_{\text {int }}\left(\vec{r}_{e}, \vec{r}_{i}\right)+U_{\text {self }}\right) \psi\left(\rho_{e}, \theta_{e}, z_{e}\right)=E_{t o t} \psi\left(\rho_{e}, \theta_{e}, z_{e}\right) .
$$

The self potential energy is only a function of the radial coordinate and is shown in Fig. 6. Notice that the self-energy for freestanding NWs has the opposite sign from that of NWs with a metallic gate. When the electron is close to the edge of the wire, the curves have a $1 /\left|R-\rho_{e}\right|$ singularity.

For our freestanding GaAs nanowire, the total energy of the ground state is shown in Fig. 7(a). The solid curves are the results from the finite element calculation, which are always smaller than the variational one. This is not surprising since the variational calculation provides an upper bound to the exact result. The curves converge for a large radius. Notice that the curve for the impurity at $\rho_{i}=0.96 R$ is distinct from the other curves even when $R$ is large. This is not so for the variational results. This difference is due to the fact that in the variational result, the trial function was such that the electron is always symmetrically localized around $\rho=0$, i.e., the electron is not able to follow the impurity when it is shifted toward the boundary of the nanowire.

In Fig. 8, we plot the relative change of the energy $E_{\text {tot }}\left(\rho_{i}\right) / E_{\text {tot }}\left(\rho_{i}=0\right)$ as a function of the impurity position using our finite element calculation. When $R$ is small (as shown by the curve for $R=2 \mathrm{~nm}$ shown in Fig. 8), the absolute value of $E_{t o t}\left(\rho_{i}\right) / E_{t o t}\left(\rho_{i}=0\right)$ stays around 1 and the slope is very small; i.e., the difference between the total energies for different impurity positions is small. However, if we increase 

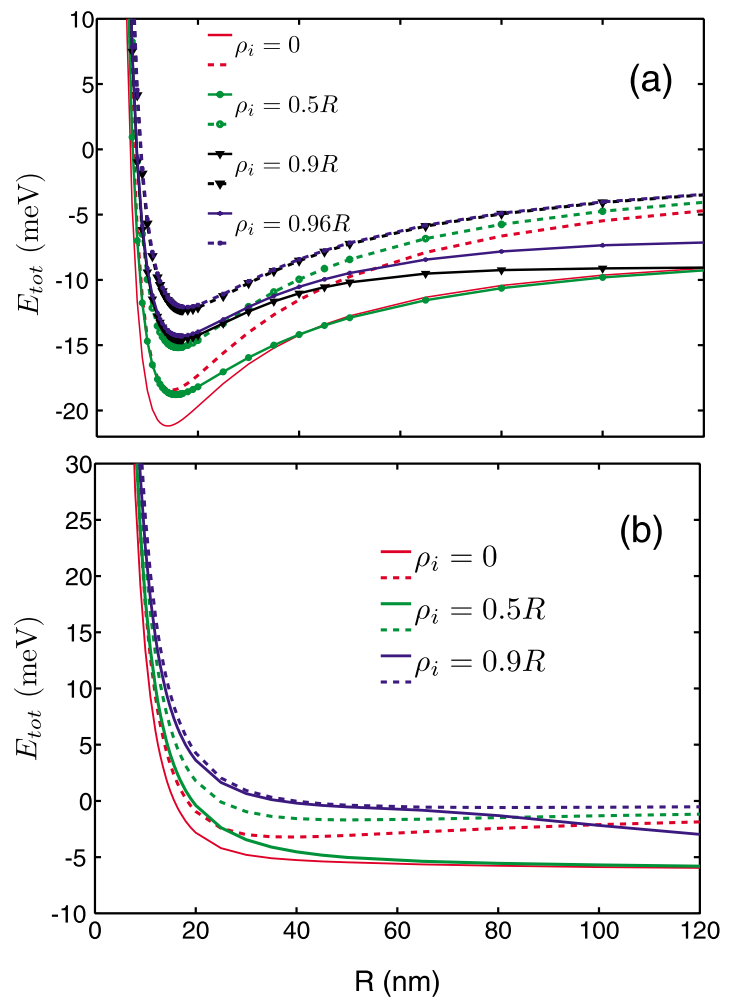

FIG. 7. (Color online) (a) Comparison between the total energy as obtained from the finite element method (solid curves) and the variational method (dash curves) for the case of freestanding GaAs nanowires. (b) The same as (a) but now for the case of a GaAs nanowire surrounded by a metallic gate.

the radius $R$, the slope will increase and the energy exhibits a larger dependence on the position of the impurity. When $R$ is beyond $5-10 \mathrm{~nm}$, the total energy becomes negative (see Fig. 7) and moving the impurity closer to the edge of the wire increases the total energy (i.e., less negative) and, consequently, the ratio $E_{t o t}\left(\rho_{i}\right) / E_{t o t}\left(\rho_{i}=0\right)$ decreases. When we continue to increase the radius $R$, the slope will decrease toward zero and the difference will become small again. These behaviors of $E_{t o t}\left(\rho_{i}\right) / E_{t o t}\left(\rho_{i}=0\right)$ can be understood as follows: (1) when $R$ is very small, the confinement energy is extremely large and dominates the total energy, the Coulomb energy coming from the electron and the impurity is negligible as compared to the confinement energy, and the confinement energy slightly depends on the impurity position, so the difference is very small when the radius $R$ is small. (2) When we increase the radius $R$, the Coulomb energy will become comparable to the confinement energy, the electron will prefer to be situated around the wire center due to the confinement, and the Coulomb part of the total energy will increase when the impurity moves from the center to the interface, so the difference between the energies of the different impurity positions will increase when moving the impurity toward the interface. (3) When $R$ is very large, the confinement energy will be negligible and the Coulomb energy will be dominant. The electron states will be similar as in a hydrogen atom for most impurity positions, so the difference in the total energy will be small again. However,

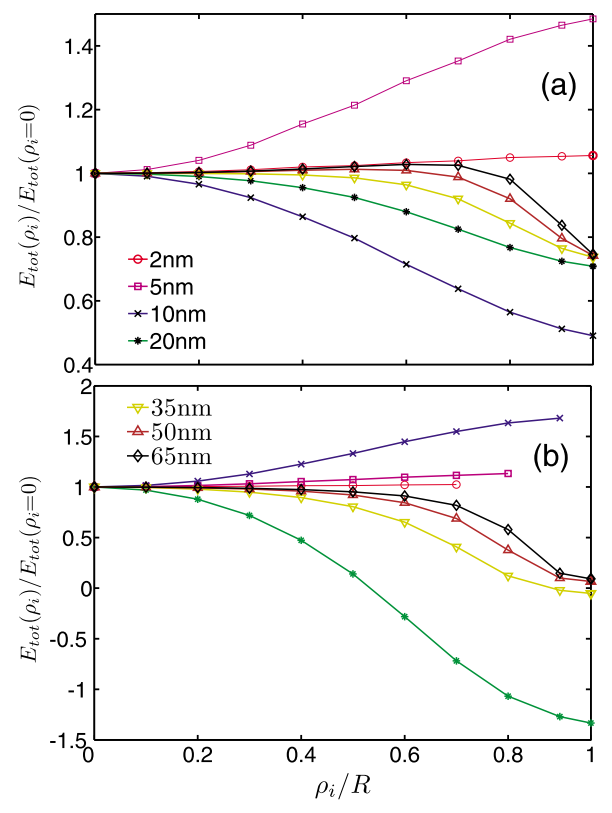

FIG. 8. (Color online) (a) The total energy as a function of the impurity position divided by the total energy for $\rho_{i}=0$ for a freestanding GaAs nanowire. (b) The same as (a) but now for the case of a GaAs nanowire surrounded by a metallic gate. Results are given for several values of the radius $R$ of the NW.

when the impurity is very close to the interface, the effect of the ionized impurity will be counteracted by the effect of its image charge, while the self-energy will increase very quickly, so the difference in the total energy will still be large. The behavior of $E_{t o t}\left(\rho_{i}\right) / E_{t o t}\left(\rho_{i}=0\right)$ for a GaAs wire surrounded by a metallic gate is similar, except that now the negative slope is formed for a larger radius $R$. When $R$ is very small and the impurity is very close to the interface, the pointlike image charge and the strong radial confinement are the response for the large number of numerical iterations needed to obtain the bound state in our finite element method.

Next, we study the binding energy using the finite element calculation. The electron energy in the absence of the impurity is first calculated by solving the following 2D Schrödinger equation:

$$
\left(-\frac{\hbar^{2}}{2 m_{e}^{*}} \nabla_{e}^{2}+U_{\text {self }}\right) \psi\left(\rho_{e}, \theta_{e}\right)=E_{\text {tot } 2} \psi\left(\rho_{e}, \theta_{e}\right) .
$$

The binding energy is defined in the following as the energy required to free the electron from its bound state:

$$
E_{\text {bind }}=E_{\text {tot2 }}-E_{\text {tot }} \text {. }
$$

The binding energy is shown in Fig. 9. In the case of a freestanding nanowire [Fig. 9(a)], we found that, qualitatively, the behavior is similar to the one obtained from the variational calculation, except for the curve with $\rho_{i}=0.96 R$. When the impurity is $0.96 R$ away from the center, the binding energy, like the total energy, will still be different from the other curves when $R$ is large. Figure 9 (b) shows the results for a GaAs wire with a metallic gate. The results are 

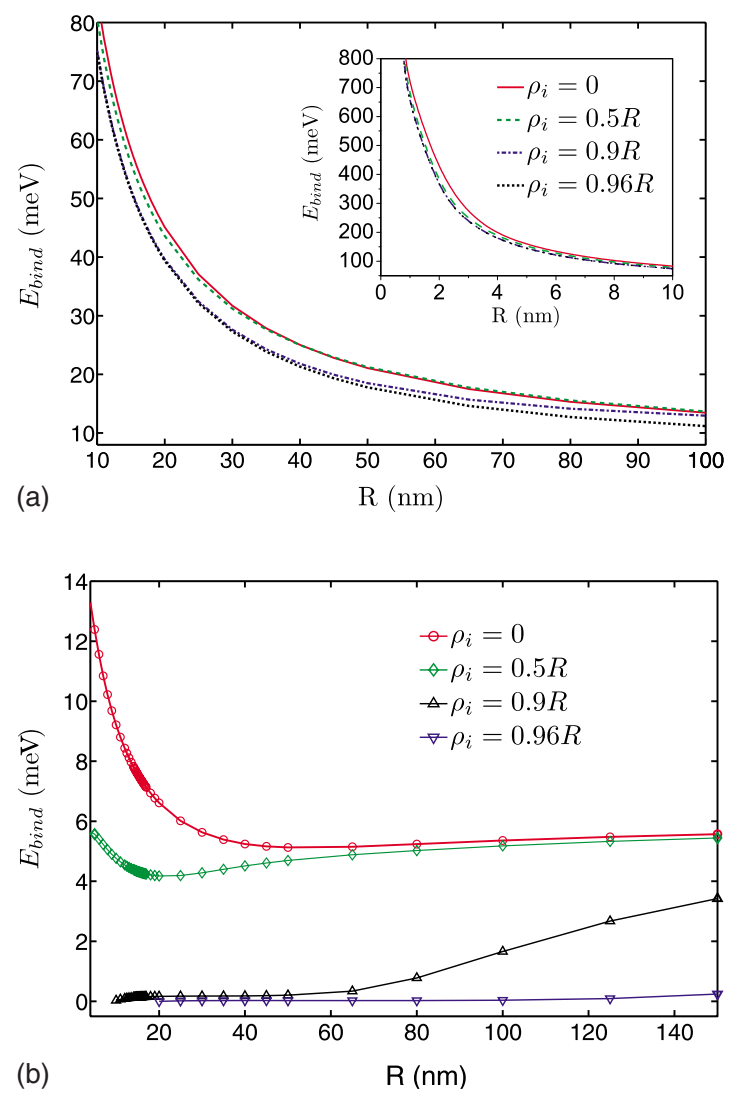

FIG. 9. (Color online) (a) The binding energy obtained from the finite element method as a function of the wire radius $R$ for a freestanding GaAs nanowire. Results are shown for four different values of impurity position $\rho_{i}$. (b) The same as (a) but now for the case of a GaAs wire with a metallic gate.

very different from those of the variational calculation. For most impurity positions, the binding energy will first decrease and then increase when we increase the radius $R$ of the wire. However, when the ionized impurity is very close to the interface, as there is only a weak bound state, the binding energy will monotonously increase (from around zero). The decreasing tendency for small $R$ can be understood as follows. When the radius is very small, since the confinement energy is very large, the electron will be compressed to move in a small area around the axis of the NW, which is also close to the impurity. Consequently, the absolute value of the Coulomb energy between the electron and the ionized impurity is large, while the self-energy and the confinement energy will be similar to the case of a wire without an impurity, and so the binding energy is large. If we increase the radius $R$, the confinement energy will be smaller and the electron will move in a larger area but still around the axis of the NW. The average distance between the electron and the impurity will also increase, so the absolute value of the Coulomb energy will be smaller. However, as the electron moves mainly around the wire axis, the change of the self-energy and the confinement energy are small, leading to a decrease of the binding energy. The increasing tendency at large $R$ (when the Coulomb energy is comparable to the confinement energy) can then be understood as follows. When the impurity is fixed at the center of the wire, the self-energy of the electron (negative) will be larger than the energy between the electron and the impurity's image. When the confinement energy is negligible, the orbit of the electron will be closer to the impurity for larger $R$ as the interaction between the electron and its image become smaller, and the binding energy will increase slightly. When the impurity is displaced from the center, the shape of the orbit of the electron will be more affected by the confinement and the attraction by the boundary due to the image charge. Consequently, the wave function will be more spread out for smaller $R$ when $R$ is not very small, as will be shown in Fig. 12. Therefore the average distance between the electron and the impurity will be larger for smaller $R$ ( $R$ should not be very small) and the Coulomb energy (also the binding energy) will be smaller, which leads to an increasing tendency of the binding energy with increasing radius $R$. However, when the radius $R$ is very large, the electron for different impurity positions will be far from the interface and the confinement energy will be almost zero, and the electron will be localized around the impurity like in a hydrogen atom. Thus, the binding energy will become independent of the impurity position for very large $R$.

We should specify that for nanowires surrounded by a metallic gate with a very small radius, the Coulomb interaction between the electron and the impurity (together with the image) will be very small and the binding energy approaches zero when the radius tends to zero, which is only shown in Fig. 9(b) when $\rho_{i} \rightarrow R$. In the case of the variational calculation, the binding energy is different from zero for most impurity positions when $R$ approaches zero. This shows clearly the limited validity of this approach. However, for the finite element method, only when the radius is extremely small, it needs a large amount of iterations to obtain the bound state, hence, we do not give the data when the radius $R$ is small or than $0.5 \mathrm{~nm}$.

\section{VALIDITY OF THE VARIATIONAL CALCULATION}

Because of its simplicity and the limited computer resources needed, the variational approach has been very popular. It provides an upper bound to the total energy. Aside from this criterion, there is no other guarantee that one is close to the exact result. The above results already indicate that the variational calculation cannot always be accurate. Therefore, a detailed comparison with the numerically exact results are important.

For the case of a semiconductor nanowire with a metallic gate, as shown in Figs. 5 and 9(b), the binding energy obtained from the variational calculation is very different from the finite element results, especially for the case when the impurity is far from the center. One reason is that in the variational calculation, we assumed the wave function of the electron to have a cylindrical symmetry. In reality, the symmetry will be destroyed when the impurity is moved away from the axis of the wire. For the case of a GaAs nanowire with a metallic gate, the attractive interaction between the electron and the interface also contributes to the destruction of the symmetry. That is an additional reason why the difference in the binding energies can be very large when the 
impurity is away from the center of the wire. For a freestanding semiconductor nanowire, as the interaction between the electron and the interface is repulsive, which partly counteracts the effect of the impurity position, the variational calculation for larger values of $R$ is much more valid. However, we cannot explain the difference of the validity for these two nanowires only on the basis of the symmetry of the wave function. As we will show later, for a GaAs nanowire with a metallic gate, the symmetry of the wave function in the lateral plane for $z=0$ will be very good when the impurity is very close to the boundary and the radius is not very large.

To further study the validity of the variational calculation, we plot in Fig. 10 the relative difference in binding energy between the finite element calculation and the variational one, $\left|E_{\text {bind,FEM }}-E_{\text {bind,var }}\right|$, as a function of the wire radius $R$. It is obvious that the relative difference for a freestanding NW is much smaller than for a NW with a metallic gate. From Fig. 10(a), we see that the difference is small when the radius is smaller than $25 \mathrm{~nm}$. Moreover, the curves for larger values of $\rho_{i}$ could be lower than the curves for smaller $\rho_{i}$, although they have worse cylindrical symmetry. For $\rho_{i}$ $>0.9 R$, the difference can be even smaller than for the case when the ionized impurity is fixed at the center. This is remarkable because the system has perfect cylindrical symmetry when the ionized impurity is fixed at the center. In order to understand this, we show the contour plot of the lateral part of the wave function in Fig. 11. As a comparison, we give also the radial part of the wave function used in the variational calculation [the two figures without impurity, the same as $\varphi\left(\rho_{e}, \theta_{e}\right)$, and proportional to $\left.\psi_{e}\left(\rho_{e}, \theta_{e}, z=0\right)\right]$ and the radial part of the wave function as obtained from the finite element method. The wave function used in the variational calculation always has a large average extension from the center of the wire. The top figures are for the case of $R$ $=15 \mathrm{~nm}$; from the last four figures, we know that the symmetry will be better and the wave function will be more spread out when the ionized impurity is closer to the interface. Here, we should point out that when the radius is $R$ $=15 \mathrm{~nm}$, since the average extension of the electron from the ionized impurity (as shown in the second figure of the top figures) is smaller for $\rho_{i}=0$ than for $\rho_{i}=0.9 R$, the difference of the previous case from the variational one will be larger, as shown in the inset of Fig. 10(a). Next, let us look at the bottom figures which are for $R=50 \mathrm{~nm}$. The difference for all values of $\rho_{i}$ with the variational wave function is large. However, due to the repulsive interaction between the electron and the interface, the wave function for a larger value of $\rho_{i}$ will be more spread out and the symmetry will be even better, so the difference for a larger value of $\rho_{i}$ is smaller. When the radius $R$ is very large, then the wave function for different values of $\rho_{i}$ will be like that of a hydrogen atom, the difference will only depend on the symmetry, and, consequently, smaller values of $\rho_{i}$ will result in smaller differences in energy, which is apparent from Fig. 10(a).

Figure 12 shows contour plots of the wave function for a GaAs nanowire with a metallic gate. When the impurity is very close to the boundary, the wave function for larger $\rho_{i}$ will have a better circular symmetry and also have a similar average extension as the variational one. The reason is that when the impurity is very close to the boundary, the Cou-
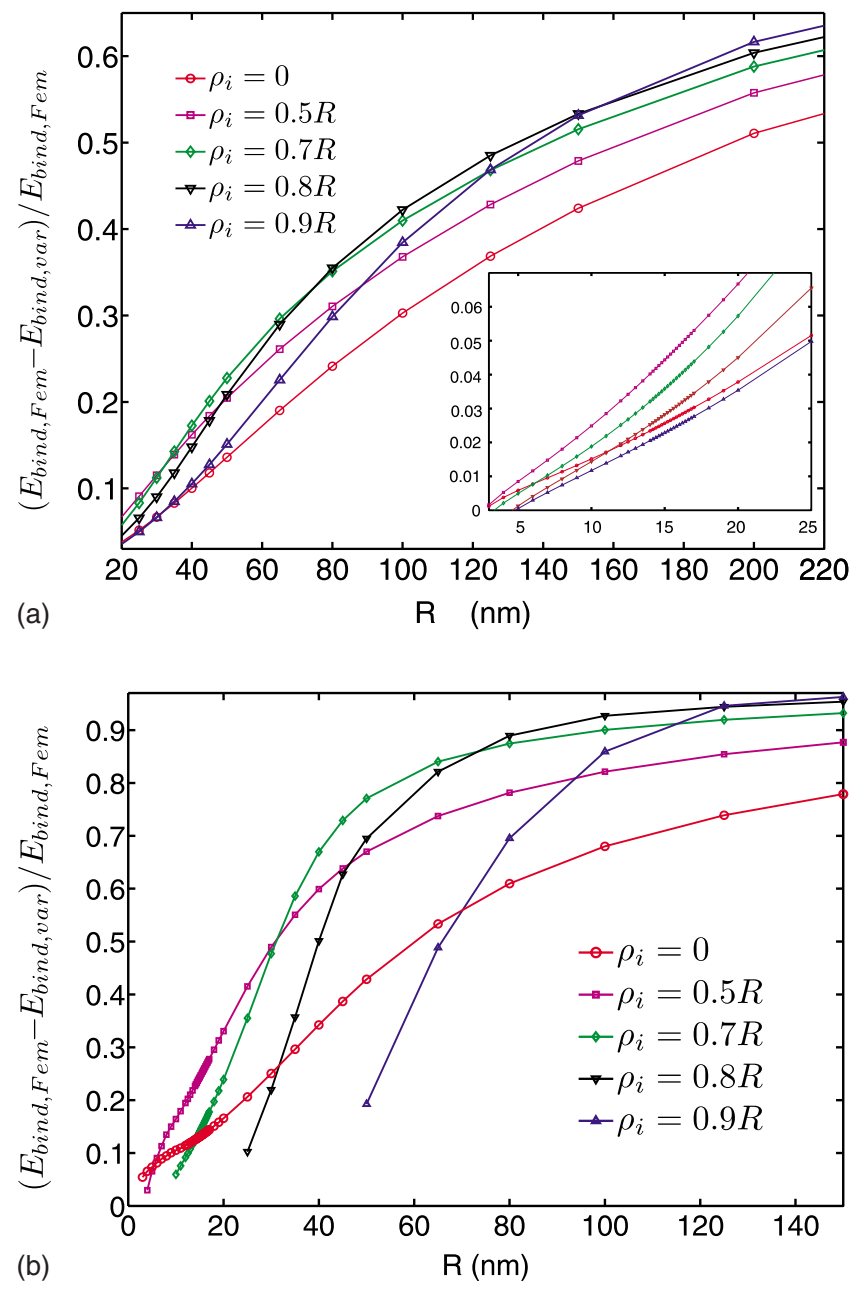

FIG. 10. (Color online) Relative difference between the binding energy obtained from the finite element calculation and the variational approach $\left|E_{\text {bind,FEM }}-E_{\text {bind,var }}\right|$ divided by the results from the finite element calculation, as a function of the wire radius $R$. (a) Freestanding GaAs nanowire. The inset is an enlargement of the curves when $R$ is smaller than $25 \mathrm{~nm}$. (b) GaAs NW surrounded by a metallic gate.

lomb energy between the electron and the impurity will counteract the Coulomb energy between the image of the impurity and the electron; the electron will only have the self-energy coming from the dielectric mismatch. Therefore, the wave function in the radial part for $z=0$ is close to the variational one. From Fig. 12, we are not able to explain why the difference in the binding energies from the two methods are so large [as shown in Fig. 10(b)]. Therefore, we calculated the average extension of the electron in the $z$ direction $\sqrt{\left\langle z^{2}\right\rangle}$, which is shown in Fig. 13. The difference in $\sqrt{\left\langle z^{2}\right\rangle}$ between the two methods is smaller for a freestanding GaAs nanowires than for a GaAs nanowire with a metallic gate. For freestanding nanowires, the difference is very small when $R<15 \mathrm{~nm}$ and becomes larger when the radius $R$ increases. However, when the impurity is very close to the boundary, the difference will still be small when $R=20 \mathrm{~nm}$, which is another reason for the unusual features shown in the inset of Fig. 10 (the binding energy difference between the 


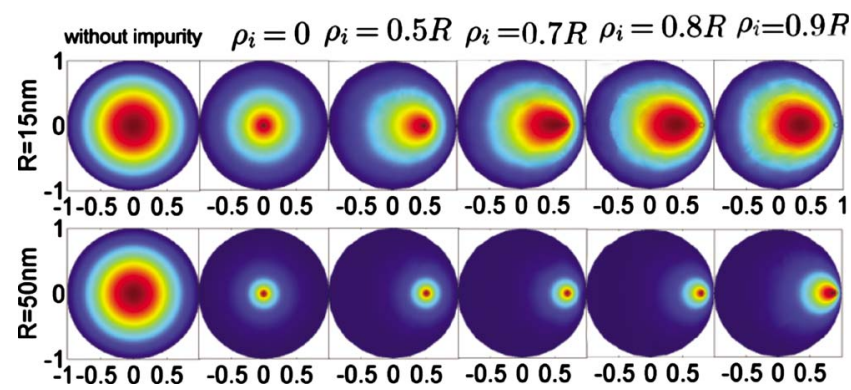

FIG. 11. (Color online) Contour plot of the ground state wave function obtained from the finite element method for a freestanding GaAs nanowire (lateral section at $z=0$ ). The two figures in the first column are the same as $\varphi\left(\rho_{e}, \theta_{e}\right)$ [radial part of the wave function, which we used in the variational calculation, proportional to $\left.\psi_{e}\left(\rho_{e}, \theta_{e}, z=0\right)\right]$. We give results for five different impurity positions and two values of the wire radius.

two methods for larger values of $\rho_{i}$ will be smaller). For a GaAs nanowire surrounded by a metallic gate, we find from Fig. 13(b) that the difference in $\sqrt{\left\langle z^{2}\right\rangle}$ from the two methods is small only when the impurity is close to the center of the wire and the radius $R$ is small. For large $\rho_{i}$, the difference in $\sqrt{\left\langle z^{2}\right\rangle}$ will be very large, which is the reason why the validity range of the variational calculation is limited. From Fig. 13, we also notice that for both types of nanowires, when the impurity position $\rho_{i}$ is not very far from the center, the average extension of the electron in the $z$ direction, $\sqrt{\left\langle z^{2}\right\rangle}$, will increase when we increase the radius of the wire $R$. However, if the impurity is very close to the boundary, $\sqrt{\left\langle z^{2}\right\rangle}$ does not increase monotonously; it will first increase, then decrease when the radius of the wire $R$ increases (see the curves for $\rho_{i}=0.9 R$ and $\left.\rho_{i}=0.96 R\right)$. However, all the curves for $\sqrt{\left\langle z^{2}\right\rangle}$ will overlap with each other when the radius is very large in both kinds of nanowires, as the ionized impurity becomes similar to a hydrogen atom.

The physical reason for the increasing tendency of $\sqrt{\left\langle z^{2}\right\rangle}$ is the same for freestanding nanowires and nanowires with a
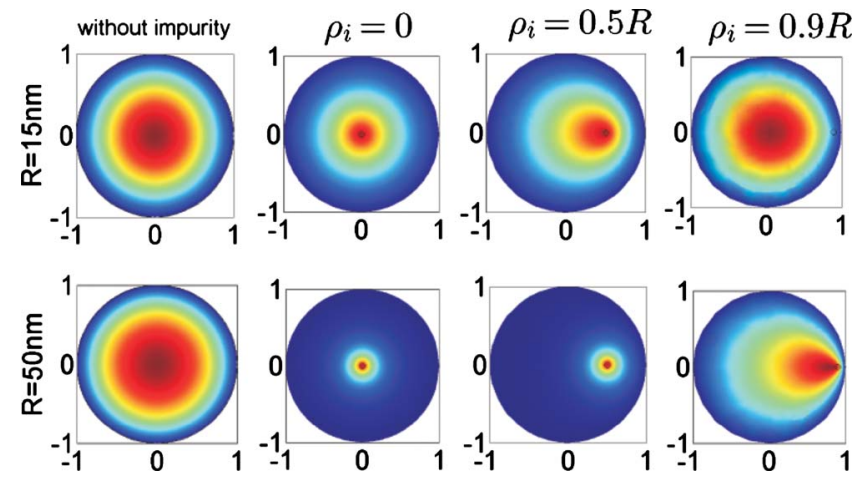

FIG. 12. (Color online) Contour plot of the ground state wave function obtained from the finite element method for a GaAs nanowire surrounded by a metallic gate (radial section for $z=0$ ). The two figures in the first column are the same as $\varphi\left(\rho_{e}, \theta_{e}\right)$ (radial part of the wave function, which we used in the variational calculation). Results are given for three different impurity positions and two values of the wire radius.

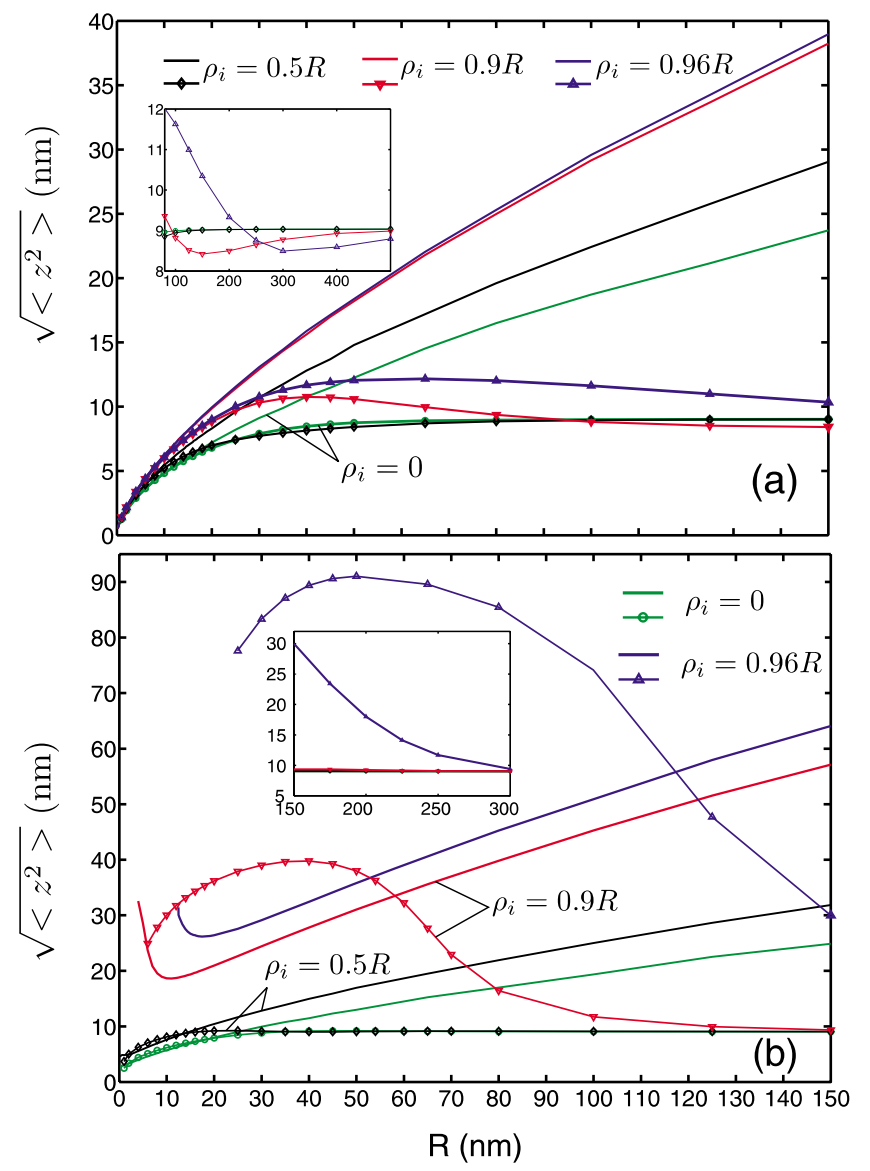

FIG. 13. (Color online) Average extension of the electron in the $z$ direction for (a) a freestanding GaAa nanowires and (b) a GaAs nanowire surrounded by a metallic gate. Solid lines are the results from the variational calculation, and the solid lines with a symbol are from FEM. We consider four different ionized impurity positions. Insets: Results for large $R$.

metallic gate, but is different for the decreasing tendency when the impurity is close to the boundary (like $\rho_{i}=0.9 R$ ). The increasing tendency can be explained with the help of Fig. 14, which is for the case of $\rho_{i}=0$. For larger $\rho_{i}$, the curves are similar, except that the well should move to the boundary. The electron can be treated as moving in an infinitely deep well due to the Coulomb interaction. For GaAs, the effective Bohr radius is around $9 \mathrm{~nm}$ in the bulk material and is proportional to the width of the well. Here, when $R$ is very small [Fig. 14(a); $R$ smaller than the effective Bohr radius], the width of the total potential well is smaller than the width in the bulk material and the electron will be closer to the impurity and has a larger Coulomb energy. When we increase the radius, the width of the well will also increase, but is still smaller than in the bulk material. Notice that Fig. 14(b) looks qualitatively the same as Fig. 14(a) but, in this case, $R=5 \mathrm{~nm}$, the width of the wire is much larger, and the Coulomb energy between the electron and the impurity will thus be smaller, resulting in an increase of $\sqrt{\left\langle z^{2}\right\rangle}$. When the radius $R$ is very large [Fig. 14(c); much larger than the effective Bohr radius of $9 \mathrm{~nm}$ ], the electron will stay in the potential well of the ionized impurity, which is similar to that 

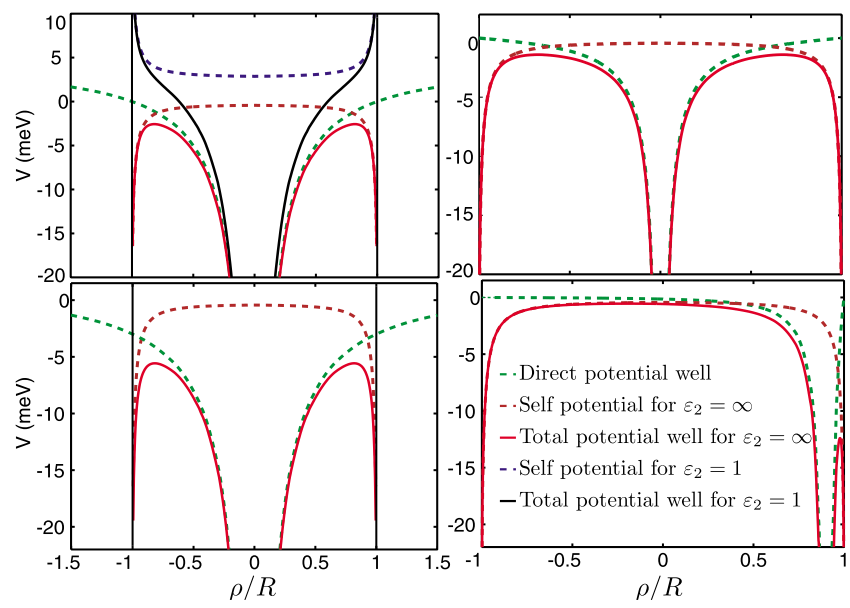

FIG. 14. (Color online) Potential well for a freestanding GaAs nanowire and a GaAs nanowire with a metallic gate: (a) $R=2 \mathrm{~nm}$, $\rho_{i}=0$; (b) $R=5 \mathrm{~nm}, \rho_{i}=0$; (c) $R=45 \mathrm{~nm}, \rho_{i}=0$, and (d) $R=30 \mathrm{~nm}$, $\rho_{i}=0.9 R$.

of an effective hydrogen atom, and thus $\sqrt{\left\langle z^{2}\right\rangle}$ will become equal to the effective Bohr radius and, consequently, all the curves will overlap. We need to point out that, as shown in Fig. 14(a), the width of the potential well for a freestanding nanowire is narrower than for a nanowire surrounded by a metallic gate and that the corresponding electron will be more spread out in the latter case.

The decreasing tendency of $\sqrt{\left\langle z^{2}\right\rangle}$ for large $\rho_{i}$ is due to the repulsive interaction from the boundary, which forces the electron to move away from the impurity. For example, when $\rho_{i}=0.9 R$ and $R=40 \mathrm{~nm}$, the distance of the impurity from the boundary is only $0.1 R=4 \mathrm{~nm}$, but the effective Bohr radius is around $9 \mathrm{~nm}$ for such a nanowire. When we continue to increase the radius $R$, the repulsive interaction will decrease, so the electron will be closer to the boundary in the radial plane (and, thus, also close to the impurity) and the Coulomb interaction between the electron and the impurity will increase, which leads to a decreasing $\sqrt{\left\langle z^{2}\right\rangle}$ with $R$.

For a GaAs nanowire surrounded by a metallic gate, we have a different situation, since the Coulomb interaction between the electron and the boundary is now attractive. As the impurity will be like an effective hydrogen atom when $R$ is very large, here we only need to confirm that there exists a range of radius where $\sqrt{\left\langle z^{2}\right\rangle}$ is very large. Actually, we can see this in Fig. 13(b), where $\sqrt{\left\langle z^{2}\right\rangle}$ for larger $\rho_{i}$ could be very large. There are two possible reasons: (1) We know that for a nanowire with a metallic gate, the self-energy is also negative and, when the impurity is very close to the boundary, the self-energy will make the Coulomb potential well much wider [superposition of two infinite deep wells as shown in
Fig. 14(d); the well will be more spread out], the Coulomb energy between the electron and the impurity will decrease, and so $\sqrt{\left\langle z^{2}\right\rangle}$ will be larger than in the bulk. (2) When the impurity is very close to the boundary, the Coulomb interaction between the electron and the image of the impurity will counteract the direct interaction between the electron and the impurity in both the lateral plane and the $z$ direction [see Fig. 14(d), where in a large region the total potential energy is close to zero]. The Coulomb interaction with the electron will be very small along the $z$ direction, leading to a large value for $\sqrt{\left\langle z^{2}\right\rangle}$.

If we extend the $R$ range as shown in the inset of Figs. 13(a) and 13(b), we find that $\sqrt{\left\langle z^{2}\right\rangle}$ tends toward a constant value $\sqrt{\left\langle z^{2}\right\rangle} \approx 9 \mathrm{~nm}$ in both cases.

\section{CONCLUSIONS}

The variational method and the finite element method were used to calculate the lowest shallow impurity states in a semiconductor nanowire with and without a metallic gate. We found that the variation of the electron total energy as function of the impurity position for two kinds of nanowires is very similar. The binding energy, on the other hand, behaves very differently for both types of nanowires. For a freestanding semiconductor nanowire, the binding energy becomes much larger due to the dielectric mismatch effect. It decreases when we increase the radius of the wire, which implies that the impurity can be ionized easier when the radius $R$ of the wire increases. The binding energy has a very weak dependence on the position of the impurity. For the nanowire with a metallic gate, the binding energy is much smaller (only several $\mathrm{meV}$ ) and the dependence on the impurity position is as important as on the radius of the wire $R$. The binding energy first decreases and then increases as we increase the wire radius $R$. When the impurity is very close to the edge of the nanowire, the binding energy approaches zero for small $R$.

The average extension in the $z$ direction $\sqrt{\left\langle z^{2}\right\rangle}$ will increase when we increase the radius $R$, which is different from the case when the impurity is very close to the boundary, where we found that $\sqrt{\left\langle z^{2}\right\rangle}$ decreases with increasing radius $R$. We found that the variational calculation is more reliable for freestanding nanowires than for NWs surrounded by a metallic gate.

\section{ACKNOWLEDGMENTS}

This work was supported by the EU-NoE: SANDiE, the Flemish Science Foundation (FWO-Vl), the Interuniversity Attraction Poles, Belgium State, Belgium Science Policy, and IMEC, vzw. 
*phymilky@gmail.com

†rancois.peeters@ua.ac.be

${ }^{1}$ Yat Li, Jie Xiang, Fang Qian, Silvija Gradecak, Yue Wu, Hao Yan, Douglas A. Blom, and Charles M. Lieber, Nano Lett. 7, 6 (2006).

${ }^{2}$ Josh Goldberger, Allon I. Hochbaum, Rong Fan, and Peidong Yang, Nano Lett. 6, 973 (2006).

${ }^{3}$ Y. Cui and C. M. Lieber, Science 291, 851 (2001).

${ }^{4}$ H. Peelaers, B. Partoens, and F. M. Peeters, Nano Lett. 6, 2781 (2006).

${ }^{5}$ H. Sellier, G. P. Lansbergen, J. Caro, S. Rogge, N. Collaert, I. Ferain, M. Jurczak, and S. Biesemans, Phys. Rev. Lett. 97, 206805 (2006).

${ }^{6}$ H. Peelaers, B. Partoens, and F. M. Peeters, Appl. Phys. Lett. 90, 263103 (2007).

${ }^{7}$ Y. Huang, X. Duan, Y. Cui, L. J. Lauhon, K. H. Kim, and C. M. Lieber, Science 302, 1313 (2003).

${ }^{8}$ H. G. Liu, K. Jun, D. A. Czarplewski, and H. G. Craighead, Nano Lett. 4, 671 (2004).

${ }^{9}$ Minhee Yun, Nosang V. Myung, Richard P. Vasquez, Choonsup Lee, Erik Menke, and Reginald M. Penner, Nano Lett. 4, 419 (2004).

${ }^{10}$ Y. Cui, Q. Wei, H. Park, and C. M. Lieber, Science 293, 1289 (2001).

${ }^{11}$ Z. Zhong, D. Wang, Y. Cui, M. W. Bockrath, and C. M. Lieber, Science 291, 1377 (2001).

${ }^{12}$ A. B. Greytak, L. J. Laudon, M. S. Gudiksen, and C. M. Lieber, Appl. Phys. Lett. 84, 4176 (2004).
${ }^{13}$ W. Lu, J. Xiang, B. P. Timko, Y. Wu, and C. M. Lieber, Proc. Natl. Acad. Sci. U.S.A. 102, 10045 (2005).

${ }^{14}$ F. Qian, S. Gradecak, Y. Li, C. Y. Wen, and C. M. Lieber, Nano Lett. 5, 2287 (2005).

${ }^{15}$ P. Mohan, J. Motohisa, and T. Fukui, Nanotechnology 16, 2903 (2005).

${ }^{16}$ P. Mohan, J. Motohisa, and T. Fukui, Appl. Phys. Lett. 88, 133105 (2006).

${ }^{17}$ P. Mohan, J. Motohisa, and T. Fukui, Appl. Phys. Lett. 88, 013110 (2006).

${ }^{18}$ A. F. Slachmuylders, B. Partoens, W. Magnus, and F. M. Peeters, Phys. Rev. B 74, 235321 (2006).

${ }^{19}$ M. Diarra, Y. M. Niquet, C. Delerue, and G. Allan, Phys. Rev. B 75, 045301 (2007).

${ }^{20}$ P. Vagner and M. Moško, J. Appl. Phys. 81, 3196 (1997).

${ }^{21}$ J. Planelles and J. L. Movilla, Phys. Rev. B 73, 235350 (2006).

${ }^{22}$ P. Vagner, D. Munzar, and M. Moško, Acta Phys. Pol. A 92, 1038 (1997).

${ }^{23}$ J. L. Movilla and J. Planelles, Phys. Rev. B 75, 195336 (2007).

${ }^{24}$ M. Mosko, D. Munzar, and P. Vagner, Phys. Rev. B 55, 15416 (1997).

${ }^{25}$ Y. M. Niquet, Nano Lett. 7, 1105 (2007).

${ }^{26}$ D. O. Demchenko and L. W. Wang, Nano Lett. 7, 3219 (2007).

${ }^{27}$ M. Royo, J. Planelles, and M. Pi, Phys. Rev. B 75, 033302 (2007).

${ }^{28}$ A. F. Slachmuylders, B. Partoens, W. Magnus, and F. M. Peeters, J. Phys.: Condens. Matter 18, 3951 (2006). 